\title{
An efficient, free-breathing protocol for MR right heart catheterization
}

\author{
Jonathan R Mazal ${ }^{1 *}$, Toby Rogers ${ }^{1}$, Anthony Z Faranesh ${ }^{1}$, Peter Kellman ${ }^{1}$, William Schenke ${ }^{1}$, Annette Stine ${ }^{1}$, \\ Laurie Grant ${ }^{1}$, Kanishka Ratnayaka ${ }^{1,2}$, Robert J Lederman ${ }^{1}$ \\ From 17th Annual SCMR Scientific Sessions \\ New Orleans, LA, USA. 16-19 January 2014
}

\section{Background}

MR right heart catheterization is a valuable diagnostic tool in patients with cardiac and pulmonary vascular disease. Many patients are dyspneic and/or require moderate sedation so MR examinations with multiple breath holds are not feasible. We describe an efficient freebreathing MR imaging protocol designed to facilitate MR catheterization in these challenging patients.

\section{Methods}

The baseline MR examination begins with cardiac localizers. At this stage it is important to verify isocenter because if the patient is too deep in the bore then femoral vascular access sheaths are inaccessible to the operator. Through-plane phase contrast (PC) scans in the ascending aorta and main pulmonary artery follow for quantification of Qp and Qs. To avoid the need to repeat long PC scans (3 averages), we first perform brief PC 'localizer' scans with a single average to confirm the selected velocityencoding factor is correct. For cardiac volumetric analysis, long and short axis views are obtained using a real-time SSFP sequence that sacrifices some spatial resolution for increased temporal resolution. Data is collected over one and a half heartbeats to ensure the entire cardiac cycle is captured. Because of the delay in image reconstruction, the short axis stack is acquired last while the operator prepares for catheterization. A dedicated user interface (Interactive Front End, Siemens; Erlangen, Germany) is used for real-time imaging during catheterization. This interface permits interactive manipulation of imaging planes, slice thickness, saturation pre-pulse, and cine loop recordings. To minimize catheterization time, reference images

Table 1 MR Right Heart Catheterization Pulse Sequence Parameters

\begin{tabular}{|c|c|c|c|c|c|c|c|}
\hline & $\begin{array}{l}\text { Tradional } \\
\text { Localizers } \\
\end{array}$ & $\begin{array}{l}\text { Multi-Slice } \\
\text { Localizer }\end{array}$ & $\begin{array}{c}\text { Localizer Phase } \\
\text { Contrast }\end{array}$ & $\begin{array}{c}\text { Diagnostic Phase } \\
\text { Contrast }\end{array}$ & $\begin{array}{l}\text { Real-Time LAX } \\
\text { Cine }\end{array}$ & $\begin{array}{l}\text { Real-Time SAX } \\
\text { Cine }\end{array}$ & $\begin{array}{l}\text { Real-Time } \\
\text { SSFP (IFE) }\end{array}$ \\
\hline TR & $2.66 \mathrm{~ms}$ & $2.81 \mathrm{~ms}$ & $4.64 \mathrm{~ms}$ & $4.84 \mathrm{~ms}$ & $2.58 \mathrm{~ms}$ & $2.46 \mathrm{~ms}$ & $2.44 \mathrm{~ms}$ \\
\hline $\mathrm{TE}$ & $1.13 \mathrm{~ms}$ & $1.23 \mathrm{~ms}$ & $2.47 \mathrm{~ms}$ & $2.64 \mathrm{~ms}$ & $1.13 \mathrm{~ms}$ & $1.04 \mathrm{~ms}$ & $1.22 \mathrm{~ms}$ \\
\hline $\begin{array}{l}\text { Matrix (Readout } \times \\
\text { Phase) }\end{array}$ & $168 \times 256$ & $160 \times 240$ & $173 \times 192$ & $256 \times 256$ & $80 \times 160$ & $80 \times 160$ & $128 \times 128$ \\
\hline Slice Thickness & $8.0 \mathrm{~mm}$ & $4.5 \mathrm{~mm}$ & $6.0 \mathrm{~mm}$ & $6.0 \mathrm{~mm}$ & $6.0 \mathrm{~mm}$ & $8.0 \mathrm{~mm}$ & $8.0 \mathrm{~mm}$ \\
\hline Flip Angle & $80^{\circ}$ & $80^{\circ}$ & $20^{\circ}$ & $20^{\circ}$ & $45^{\circ}$ & $45^{\circ}$ & $45^{\circ}$ \\
\hline $\begin{array}{l}\text { Acceleration } \\
\text { Factor }\end{array}$ & 2 & 2 & 2 & 2 & 4 & 4 & 4 \\
\hline Cardiac Gating & None & None & ECG/Retro & ECG/Retro & $\begin{array}{l}\text { ECG/Trigger } \\
\text { (1.5 Beats) }\end{array}$ & $\begin{array}{l}\text { ECG/Trigger } \\
\text { (1.5 Beats) }\end{array}$ & None \\
\hline Scan Times & $10 \mathrm{sec}$ & $35 \mathrm{sec}$ & $23 \mathrm{sec}$ & 2-3 min each & $3 \mathrm{sec}$ & $35 \mathrm{sec}$ & $\begin{array}{c}\text { Infinite } \\
\text { Measurements }\end{array}$ \\
\hline
\end{tabular}

\footnotetext{
${ }^{1}$ National, Heart, Lung, and Blood Institute - NIH, Bethesda, Maryland, USA
}

Full list of author information is available at the end of the article 


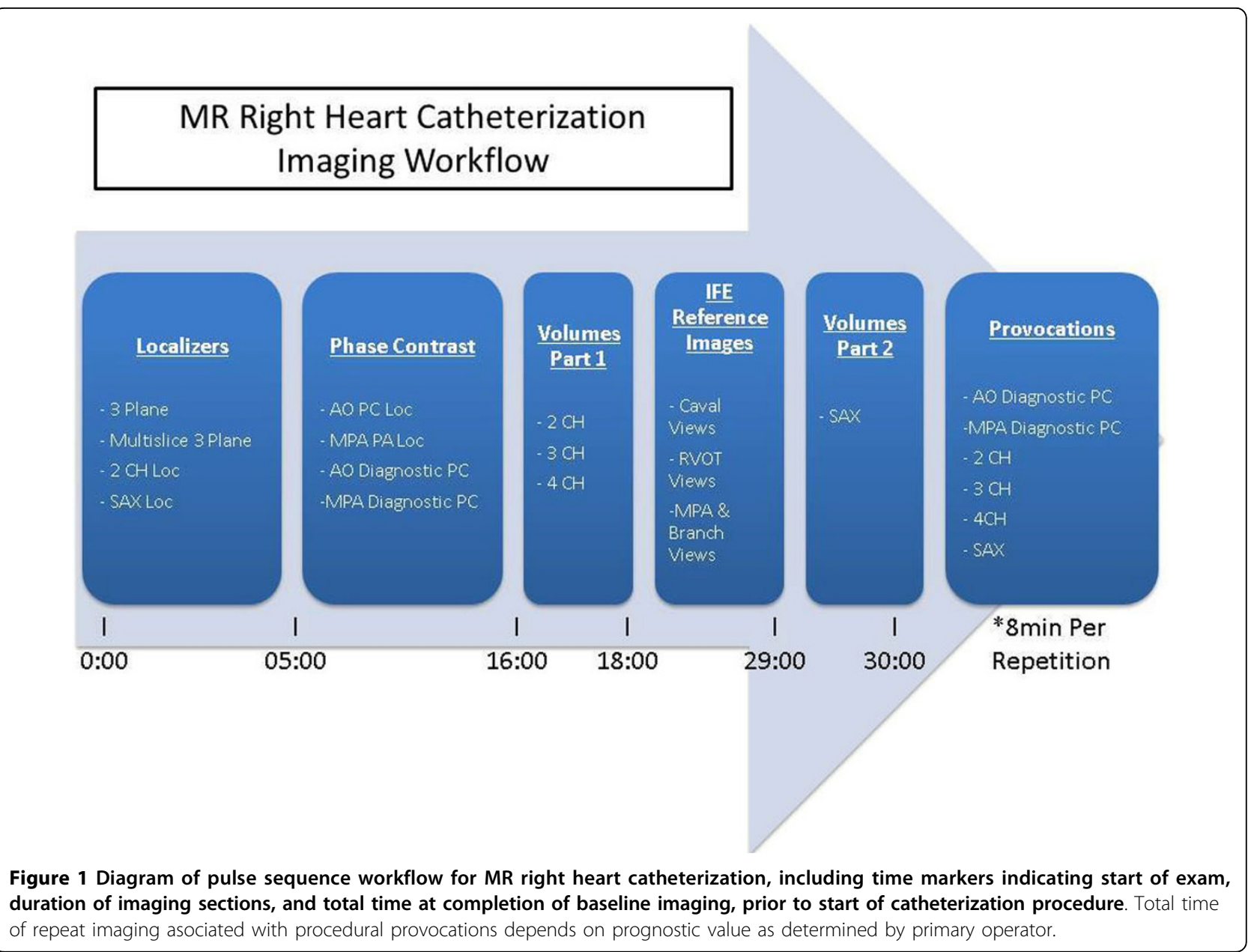

consisting of orthogonal views of the superior and inferior vena cavae, the right ventricular outflow tract, and the main and branch pulmonary arteries are prescribed prior to commencing catheterization. If additional physiological provocations during catheterization are indicated, then the baseline imaging sequences are repeated during each provocation via automatic propagation of the sequence parameters.

\section{Results}

Acquisition order was optimized to shorten scan times by enhancing scanner duty cycle. With practice we reduced the baseline MR examination time from 50 minutes to 30 minutes. With parameter duplication, subsequent scan time during physiological provocations was reduced to 8 minutes per provocation. We are now able to perform baseline MR examination, right heart catheterization and multiple physiological provocations.

\section{Conclusions}

This free-breathing MR examination protocol enables MR catheterization with multiple physiological provocations to be performed in patients with dyspnea and/or under moderate conscious sedation.

\section{Funding}

This work is supported by the Division of Intramural Research (Z01-HL005062-08, Z01-HL006039-01), National Heart Lung and Blood Institute, National Institutes of Health, USA.

\section{Authors' details}

${ }^{1}$ National, Heart, Lung, and Blood Institute - NIH, Bethesda, Maryland, USA. ${ }^{2}$ Children's National Medical Center, Washington DC, District of Columbia, USA.

Published: 16 January 2014

doi:10.1186/1532-429X-16-S1-T5

Cite this article as: Mazal et al:: An efficient, free-breathing protocol for MR right heart catheterization. Journal of Cardiovascular Magnetic Resonance 2014 16(Suppl 1):T5. 